\title{
UBV PHOTOMETRY OF THE OPEN CLUSTERS NGC 6604 AND NGC 6704
}

\author{
Doug Forbes and David L. DuPuY \\ Burke-Gaffney Observatory, Saint Mary's University, Halifax, Nova Scotia B3H 3C3, Canada \\ Received 19 July 1977; revised 12 December 1977
}

\begin{abstract}
The open clusters NGC 6604 and NGC 6704 have been studied with UBV photoelectric and photographic photometry, resulting in improved distance estimates and color-magnitude diagrams. NGC 6604 is a very young $\left(\sim 4 \times 10^{6} \mathrm{yr}\right)$ cluster with 30 probable members. The color-magnitude diagram shows a bright, well-defined main sequence; the central star of the cluster, HD 167971, is an O8f supergiant which appears to be slightly evolved. The fainter stars with $(B-V)_{0} \geq 0.0$ appear to lie above the zero-age main sequence and may be pre-main-sequence objects still in the process of gravitational contraction. This result must be confirmed because of uncertainty in the photometric calibration near $V=16$ mag. NGC 6704 is a moderately young $\left(\sim 2 \times 10^{7} \mathrm{yr}\right)$ cluster with 17 probable members observed, including the eclipsing binary FN Sct. The color-magnitude diagram shows a fairly welldefined main sequence, but no giant members are observed.
\end{abstract}

\section{INTRODUCTION}

A photometric investigation of several open clusters in the region of galactic longitudes $l=0^{\circ}-90^{\circ}$ has been initiated to obtain improved distance estimates and color-magnitude diagrams. These clusters have been chosen on the basis of few previously published distance estimates or other data, potential youth, and the possible presence of unstable stars: This paper contains the results of $U B V$ photoelectric and photographic photometry of two clusters.

NGC 6604 , located at $18^{\mathrm{h}} 15^{\mathrm{m}} 3,-12^{\circ}, 15^{\prime}(1950) ; l$ $=18^{\circ} .3, b=+1^{\circ} 7$ (1950), belongs to class I3p (Ruprecht 1966). The earliest spectral types are 05-7 (Moffat and Vogt 1975) and previous distance estimates range from 0.7 to $4.4 \mathrm{kpc}$ (Alter, Ruprecht, and Vanysek 1970). The cluster is associated with the $\mathrm{H}$ II region RCW 167 (Rodgers, Campbell, and Whiteoak 1960). The only photometry of which we are aware is the $U B V-\mathrm{H} \beta$ study of Moffat and Vogt (1975), who derived a distance of $1.64 \mathrm{kpc}$ and reddening $E(B-V)$ of $1.01 \mathrm{mag}$.

NGC 6704 is located at $18^{\mathrm{h}} 48^{\mathrm{m}} \cdot 2,-05^{\circ} 16^{\prime}$ (1950); $l=28^{\circ} .2, b=-2^{\circ} .2(1950)$ and belongs to class $\mathrm{I} 3 \mathrm{~m}$ (Ruprecht 1966). Earliest spectral types are $\sim$ B2 (Grubbisich 1965) and distance estimates vary from 1.4 to $3.2 \mathrm{kpc}$ (Alter, Ruprecht, and Vanysek 1970). The eclipsing binary system FN Sct (Bakos 1950) is a probable member of the cluster. The only known photometry is that of Grubbisich (1965) who obtained a distance of $1.81 \mathrm{kpc}$ and a reddening $E(G-R)=1.81$ mag from a photographic study in the $R G U$ system.

\section{OBSERVATIONS}

The clusters were observed with the University of Toronto 60-cm telescope at the Las Campanas Observatory by Dr. G. A. Welch in June 1975 and by one of us (D.F.) in July 1976. For all photoelectric observations, an RCA 1 P21 photomultiplier tube cooled by dry ice was used with SSR pulse-counting equipment and standard $U B V$ filters.

$U B V$ values of stars observed by Landolt (1973) were used to determine extinction coefficients and transformation equations for each night's observations, following the general technique of Hardie (1962). The visual extinction was no larger than $k_{v}=0.14$ mag for all observations. A number of stars in each cluster field were selected as photoelectric sequence stars, with a range in $V$ of 9-15.5 mag. The adopted mean $U B V$ magnitudes for these stars were formed by combining the photoelectric values derived from the 1975 observations of Welch with the 1976 observations. These $U B V$ data are given in Table I. The average standard deviations of the photoelectric magnitudes in each cluster are $0.02 \mathrm{mag}$ in $V$ and $(B-V)$ and $0.04 \mathrm{mag}$ in $(U-B)$.

Photographic plates were obtained in 1975 and 1976. Kodak spectroscopic plates $103 \mathrm{a}-\mathrm{O}$ and $103 \mathrm{a}-\mathrm{D}$ were used with UG-2, GG-385, and RG-495 filters for standard plate/filter combinations. Approximate exposure times were $1 \mathrm{~h}$ for $V$ and $B$ plates and $2 \mathrm{~h}$ for $U$ plates. A $B$ plate of each cluster is shown in Figs. 1 and 2, with identification of stars observed photoelectrically (letters) and stars measured photographically (numbers).

Three plates in each of $U, B$, and $V$ of both clusters were measured with an Astro-Mechanics iris photometer; the faintest stars measured $(V \sim 16)$ were well above the plate limit, except on $U$ plates. Approximately 100 stars were measured within a circle of 10 arcmin concentric to the cluster center on each of the 18 plates.

A calibration curve for each plate was fitted by eye to the photoelectric and iris data, using the $U B V$ values from Table I. The smaller number of usable photoelectric sequence stars for NGC 6704 made it desirable to supplement this list with additional $U B V$ data for several 266
Astron. J. 83 (3), Mar. 1978
0004-6256/78/8303-0266\$00.90
(C) 1978 Am. Astron. Soc.
266 


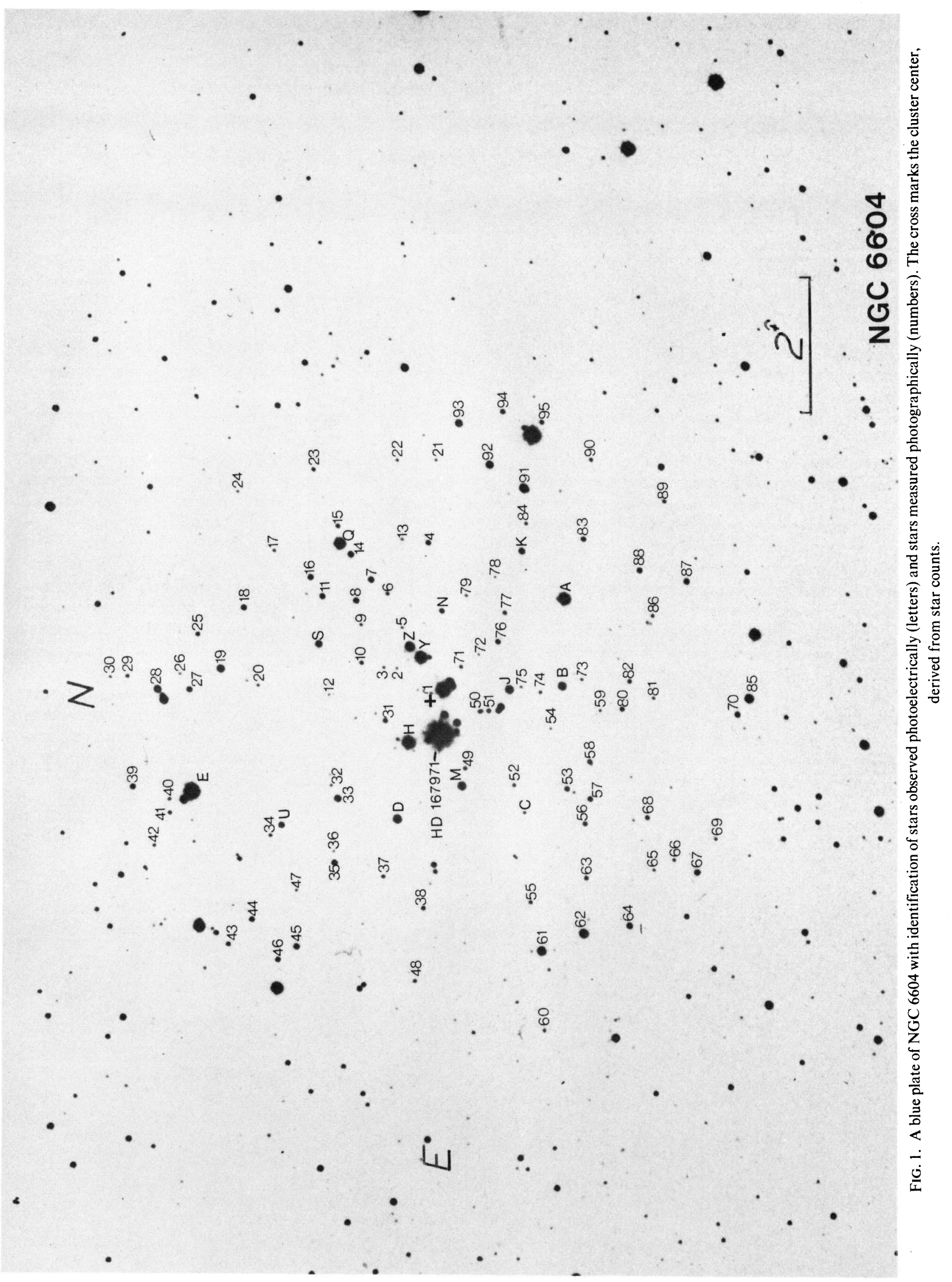




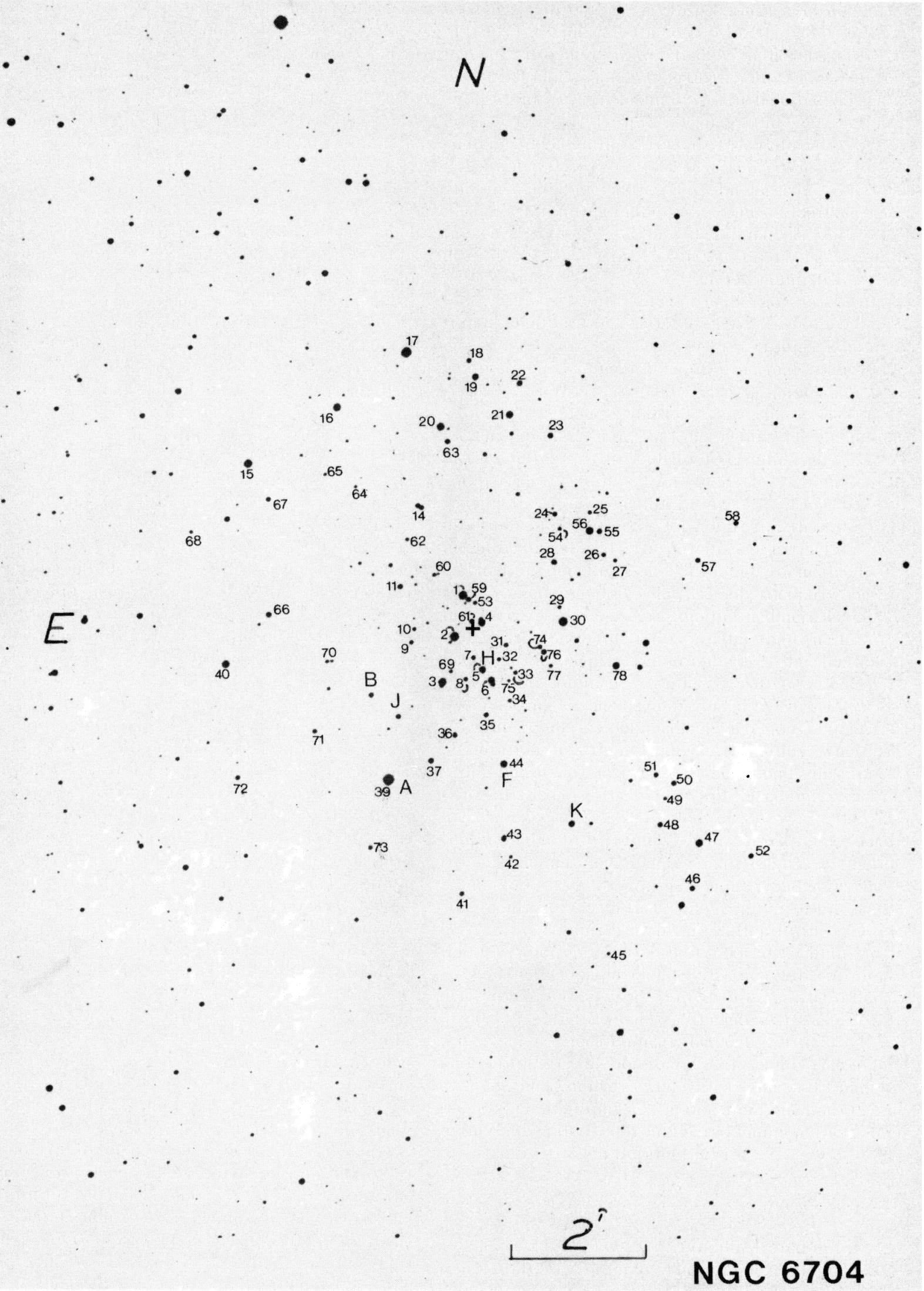

FIG. 2. A blue plate of NGC 6704, as for Fig. 1. 
TABLE I. Photoelectric sequence stars.

\begin{tabular}{lcrrrr}
\hline \hline & Star & $U-B$ & $B-V$ & $V$ & $\mathrm{n}$ \\
\hline \multirow{2}{*}{ NGC 6604 } & $\mathrm{A}$ & -0.44 & 0.75 & 10.48 & 5 \\
& $\mathrm{~B}$ & +1.76 & 1.90 & 12.51 & 4 \\
& $\mathrm{C}$ & +0.83 & 0.73 & 15.43 & 1 \\
& $\mathrm{D}$ & -0.38 & 0.70 & 12.10 & 6 \\
& $\mathrm{E}$ & -0.61 & 0.60 & 9.19 & 2 \\
& $\mathrm{G}$ & -0.31 & 0.18 & 7.94 & 2 \\
& $\mathrm{H}$ & -0.53 & 0.60 & 10.15 & 5 \\
& $\mathrm{~J}$ & -0.27 & 0.69 & 12.21 & 4 \\
& $\mathrm{~K}$ & +0.19 & 0.52 & 13.82 & 1 \\
& $\mathrm{M}$ & -0.44 & 0.69 & 12.16 & 2 \\
& $\mathrm{~N}$ & -1.14 & 1.67 & 13.83 & 1 \\
& $\mathrm{Q}$ & -0.36 & 0.84 & 10.78 & 1 \\
& $\mathrm{~S}$ & $+3.41:$ & $2.26:$ & 13.20 & 1 \\
& $\mathrm{U}$ & -0.09 & 0.76 & 13.61 & 2 \\
& $\mathrm{X}$ & -0.38 & 0.77 & 7.48 & 3 \\
& $\mathrm{Y}$ & -0.50 & 0.63 & 10.31 & 2 \\
& $\mathrm{Z}$ & -0.44 & 0.67 & 11.58 & 2 \\
& & & & & \\
& $\mathrm{~A}$ & +2.15 & 2.04 & 11.35 & 4 \\
$\mathrm{NGC} 6704$ & $\mathrm{~B}$ & +0.65 & 1.14 & 14.40 & 3 \\
& $\mathrm{~F}$ & +0.80 & 0.83 & 13.18 & 4 \\
& $\mathrm{H}$ & +0.15 & 0.64 & 13.24 & 4 \\
& $\mathrm{~J}$ & +1.33 & 1.84 & 14.40 & 2 \\
& $\mathrm{~K}$ & $\ldots$ & 2.64 & 13.66 & 1 \\
\hline \hline
\end{tabular}

stars observed by Grubbisich (1965). The transformation from the $R G U$ values given by Grubbisich to the $U B V$ system was done using the equations of Steinlin (1968); the agreement between the photoelectrically derived $U B V$ magnitudes and those transformed from $R G U$ for several "check" stars was within \pm 0.03 mag (s.d.).

The photographically derived values of the photoelectric stars were plotted versus instrumental colors and magnitudes; linear transformations to the $U B V$ system were derived. No significant trends with color or magnitude were apparent in the residuals of photoelectric minus photographic measures. Instrumental magnitudes of the program stars were averaged over the three plates of similar color and transformed to $U B V$. The average standard deviations of the transformations for both clusters are $0.04 \mathrm{mag}$ in $V$ and $(B-V)$ and $0.07 \mathrm{mag}$ in $(U-B)$. [The standard deviation of the mean $V$ magnitude of several stars in NGC 6604 was significantly greater than the average deviation; these stars are noted as possible variables in Table II. The General Catalogue of.Variables Stars (Kukarkin et al. 1969 and supplements) was searched for known variables; none were found within 30 arcmin of either cluster.] The $U B V$ data for program stars in each cluster are given in Tables II and III.

\section{ANALYSIS}

\section{a) Star Counts}

Counts were made of stars brighter than $B=15.5$ on prints of $B$ plates of both clusters in order to estimate their extent and to serve as a check on the reality of the clusters. The cluster fields were divided into quadrants centered on an estimate of the cluster centers. Each field was further divided into six concentric rings, with each ring 1.25 arcmin in radius larger than the preceding one.
Star counts were also made in eight areas of $1 \mathrm{sq}$ arcmin located randomly about the cluster at an average radial distance of $10 \mathrm{arcmin}$ (i.e., outside the sixth ring) to determine the projected density of the background.

Figure 3 illustrates the results of these counts, showing the projected density (stars/sq arcmin) for each of the six rings, for each of the four quadrants. The mean background density, averaged over the eight areas, is indicated by the dotted line. There are significant concentrations of stars above the background level with a radius of about 3 arcmin around the centers of each cluster.

\section{b) Reddening}

A preliminary estimate of the interstellar reddening was made for each cluster with a sliding fit (Becker and Stock 1954) of the two-color data to the locus of intrinsic colors of main-sequence stars (Allen 1973). The slope of the reddening line was taken as $E(U-B)=0.72 E(B$ $-V$ ). The $Q$ technique of Johnson and Morgan (1953) was also used to estimate reddening, where

$$
Q=(U-B)-[E(U-B) / E(B-V)](B-V),
$$

and the intrinsic color indices are given by $(B-V)_{0}=$ $0.332 Q$ and $(B-V)_{0}=0.270(U-B)_{0}($ Johnson 1958).

A mean color excess was found from those cluster photoelectric sequence stars with calculated color excess

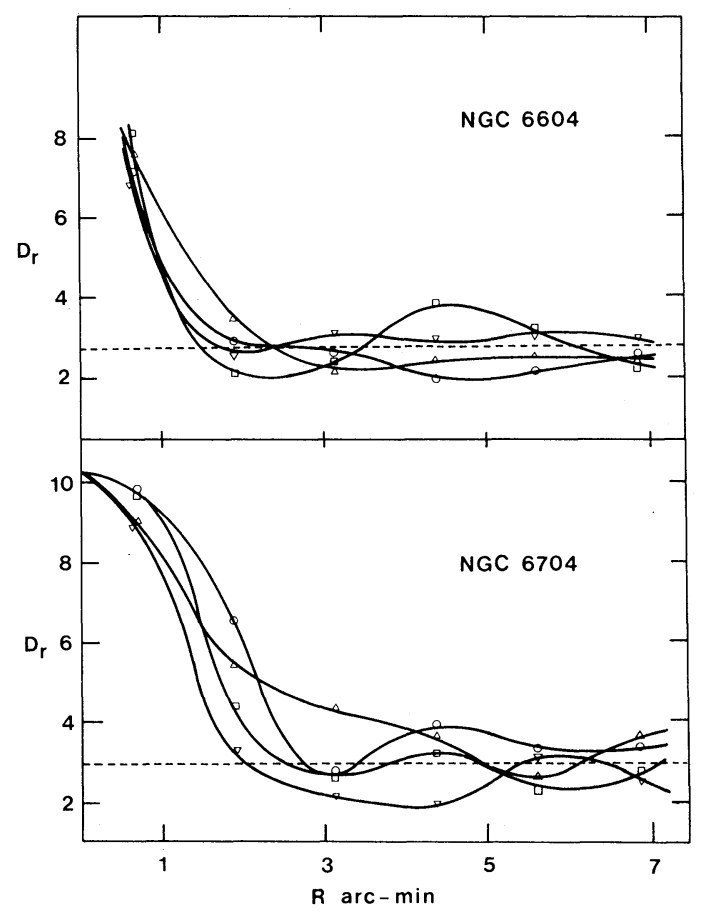

FIG. 3. Projected density of stars $D_{r}$ (stars/sq arcmin), for stars brighter than $B=15.5$, versus radial distance $R$ from cluster center. Dashed line indicates background. Results are shown for four quadrants. 